\title{
High serum concentrations of surfactant protein $A$ in usual interstitial pneumonia compared with non-specific interstitial pneumonia
}

\author{
H Ishii, H Mukae, J Kadota, H Kaida, T Nagata, K Abe, S Matsukura, S Kohno
}

Thorax 2003;58:52-57

See end of article for authors' affiliations

Correspondence to:

Correspondence to:
Dr H Mukae, Second Department of Internal

Medicine, Nagasaki

University School of

Medicine, 1-7-1 Sakamoto,

Nagasaki 852-8501,

Japan;

hmukae@net.nagasaki-u.ac.jp

Revised version received 28 August 2002

Accepted for publication

2 September 2002
Background: The pathological diagnosis of interstitial lung diseases (ILD) by surgical lung biopsy is important for clinical decision making. There is a need, however, to use serum markers for differentiating usual interstitial pneumonia (UIP) from other ILD. Surfactant protein (SP)-A, SP-D, KL-6, sialyl SSEA-1 (SLX), and sialyl Lewis ${ }^{a}$ (CA 19-9) are useful markers for the diagnosis and evaluation of activity of ILD. We have investigated the usefulness of these proteins as markers of UIP.

Methods: Serum and bronchoalveolar lavage (BAL) fluid levels of the above five markers were measured in 57 patients with various forms of ILD 119 with UIP, 12 with non-specific interstitial pneumonia (NSIP), eight with bronchiolitis obliterans organising pneumonia (BOOP), and 10 with sarcoidosis), eight patients with the control disease (diffuse panbronchiolitis (DPB)), and nine healthy volunteers. Results: Serum levels of SP-A, SP-D, and KL-6 in patients with UIP and NSIP were significantly higher than in healthy volunteers. In particular, the serum levels of SP-A in patients with UIP were significantly higher than in patients with NSIP ( $p<0.0001$, mean difference $-58.3 \mathrm{ng} / \mathrm{ml}, 95 \%$ confidence interval -81.6 to -35.0$)$, and BAL fluid levels of SP-D in patients with UIP were significantly lower than in patients with NSIP ( $p=0.01$, mean difference $322.4 \mathrm{ng} / \mathrm{ml}, 95 \%$ confidence interval 79.3 to 565.5 ). Conclusion: Serum SP-A levels may be clinically useful as a biomarker to differentiate between UIP and NSIP.
$\mathrm{N}$ on-specific interstitial pneumonia (NSIP) was first defined by Katzenstein and Fiorelli in 1994, ${ }^{1}$ and the name has gained broad acceptance as noted by Travis et $a l^{2}$ Subsequent reports indicated that patients with cellular NSIP had excellent long term prognosis, while the majority of patients with fibrotic NSIP died mostly within 5-10 years of diagnosis. ${ }^{2}$ However, the prognosis is generally good, and the response to corticosteroids and immunosuppressants is also good in patients with NSIP compared with patients with usual interstitial pneumonia (UIP)..$^{3-9}$ Although surgical (open or thoracoscopic) lung biopsy has traditionally been the "gold standard" for the pathological diagnosis of interstitial lung diseases (ILD) and is clinically relevant for selecting appropriate treatment, ${ }^{10}$ it is a relatively invasive examination, especially for patients with advanced ILD.

Surfactant protein (SP)-A, SP-D, KL-6, sialyl SSEA-1 (SLX) and sialyl Lewis ${ }^{\text {a }}$ (CA19-9) are useful markers for confirming the diagnosis and evaluation of disease activity of various ILD. SP-A and SP-D, which are lung specific proteins, belong to a subgroup of the C-type lectin superfamily, along with mannose binding lectin and collectin-43. They are mainly produced by type II pneumocytes and Clara cells within the lung, and play important roles in the lung's innate immune system. ${ }^{11-14}$ McCormack and colleagues ${ }^{15}$ showed that the levels of SP-A/phospholipid in bronchoalveolar lavage (BAL) fluid could predict survival in patients with idiopathic pulmonary fibrosis (IPF). It has also been reported that serum levels of SP-A and SP-D are significantly higher in patients with IPF than in healthy volunteers, and that a combination of the assays for SP-A and SP-D may be helpful in predicting the outcome of patients with IPF. ${ }^{12}$

Common features of the carbohydrate antigens KL-6, SLX, and CA19-9 include their high molecular weight and the fact that they are mucin-like glycoproteins. ${ }^{16}$ These three markers were initially established as serum tumour markers; KL- 6 and SLX have been used as markers for pulmonary adenocarci- noma and CA19-9 for gastrointestinal malignancies, particularly pancreatic carcinoma. ${ }^{17-19}$ However, the serum levels of these three markers are also raised in patients with non-malignant lung diseases such as interstitial pneumonia. ${ }^{20-23}$ Serum and BAL fluid levels of KL-6, first described by Kohno et al in 1985, ${ }^{19}$ are raised in patients with interstitial pneumonia. ${ }^{22-24}$ Several investigators have also reported that KL-6 is a useful serum marker for confirming the diagnosis and for long term management of various ILD. ${ }^{162324}$ Moreover, Yokoyama et al ${ }^{16}$ reported that the serum level of KL-6 was the best marker for interstitial pneumonia among carbohydrate antigens.

At present it is difficult to establish the pathological diagnosis in patients with ILD without invasive examination such as surgical lung biopsy. We have measured the levels of SP-A, SP-D, KL-6, SLX and CA19-9 in both serum and BAL fluid samples obtained from patients with various ILD to determine their usefulness as markers for predicting the pathological diagnosis.

\section{METHODS}

\section{Study population}

The subjects of this study were patients and healthy volunteers enrolled in the hospitals of Nagasaki University School of Medicine and Miyazaki Medical College. They included 19 patients with UIP, 12 with NSIP, eight with bronchiolitis obliterans organising pneumonia (BOOP), 10 with sarcoidosis, eight with diffuse panbronchiolitis (DPB), and nine healthy volunteers (seven men and two women, all nonsmokers, mean (SD) age 24.4 (4.7) years). None of these patients had received steroids or erythromycin treatment at the time of clinical sample collection. Patients with cancer in any organ and those suspected to have malignancy were excluded from the study, and no malignancy was detected in any patient during the study. Lung diseases were diagnosed as described below. 
Table 1 Total and differential cell counts in BAL fluid

\begin{tabular}{|c|c|c|c|c|c|c|c|}
\hline & $\mathrm{n}$ & $\begin{array}{l}\text { Total cells } \\
\left(10^{5} / \mathrm{ml}\right)^{*}\end{array}$ & $\begin{array}{l}\text { Macrophages } \\
(\%)^{*}\end{array}$ & $\begin{array}{l}\text { s Lymphocytes } \\
(\%)^{*}\end{array}$ & $\begin{array}{l}\text { Neutrophils } \\
(\%)^{*}\end{array}$ & $\begin{array}{l}\text { Eosinophils } \\
(\%)\end{array}$ & $\begin{array}{l}\mathrm{CD} 4 / \mathrm{CD} 8 \\
\text { ratio** }\end{array}$ \\
\hline Patients & 57 & & & & & & \\
\hline UIP & 19 & $4.5(1.8)$ & 73.8 (14.4) & $13.1(9.7)$ & $7.3(7.3)$ & $5.1(8.2)$ & $1.50(1.27)$ \\
\hline NSIP & 12 & $3.5(1.1)$ & $58.5(20.8)$ & $35.9(20.3)$ & $2.8(2.8)$ & $2.3(1.6)$ & $0.67(0.78)$ \\
\hline BOOP & 8 & $5.7(2.5)$ & $57.6(25.5)$ & $32.2(25.8)$ & $7.1(7.3)$ & $6.9(10.7)$ & $0.94(0.68)$ \\
\hline Sarcoidosis & 10 & $3.4(2.9)$ & $56.6(16.1)$ & 39.8 (17.7) & $3.1(2.9)$ & $0.6(1.1)$ & $3.17(2.22)$ \\
\hline DPB & 8 & $9.2(4.4)$ & $19.3(20.6)$ & $8.5(8.0)$ & $71.2(27.9)$ & $0.3(0.3)$ & $0.92(0.65)$ \\
\hline $\begin{array}{l}\text { Healthy } \\
\text { volunteers }\end{array}$ & 9 & $1.4(0.9)$ & $86.5(8.0)$ & $12.3(7.8)$ & 1.5 (1.1) & $1.0(1.7)$ & $1.21(0.48)$ \\
\hline \multicolumn{8}{|c|}{$\begin{array}{l}\text { Data are expressed as mean }(S D) \text {. } \\
\text { UIP=usual interstitial pneumonia; } N S I P=n o n-s p e c i f i c \text { interstitial pneumonia; } B O O P=\text { bronchiolitis obliterans } \\
\text { organising pneumonia; } D P B=\text { diffuse panbronchiolitis. } \\
{ }^{*} p<0.0001 \text { for the overall comparison of all six subject groups; }{ }^{* *} p=0.0005 \text { for the overall comparison o } \\
\text { all six subject groups. }\end{array}$} \\
\hline
\end{tabular}

Usual interstitial pneumonia (UIP)

Nineteen patients with idiopathic UIP (16 men and three women; eight current smokers, four ex-smokers, and seven non-smokers) of mean (SD) age 59.2 (9.8) years (range 34-68) were selected for the study. Patients with UIP associated with collagen vascular diseases (CVD) were excluded as these are a different group of patients who usually have a better prognosis. The diagnosis was pathologically confirmed by open lung biopsy (OLB) or video assisted thoracoscopic surgery (VATS) in all patients. The mean (SD) percentage vital capacity (\%VC) was 86.2 (22.1)\% (range 55.8-117.0) and mean arterial oxygen tension $\left(\mathrm{PaO}_{2}\right)$ measured while breathing room air was 10.9 (1.8) kPa (range 7.3-13.8).

Non-specific interstitial pneumonia (NSIP)

Twelve patients with idiopathic NSIP (four men and eight women; two current smokers, one ex-smoker, and nine non-smokers) of mean (SD) age 53.2 (11.7) years (range 28-71) were selected. Patients with NSIP associated with CVD were excluded. The diagnosis was pathologically confirmed by OLB or VATS in all patients. The mean (SD) \%VC was 83.3 (15.6)\% (range 56.8-106.2) and mean $\mathrm{PaO}_{2}$ while breathing room air was 10.8 (1.2) $\mathrm{kPa}$ (range 8.1-11.9).

Bronchiolitis obliterans organising pneumonia (BOOP) Eight patients with BOOP (four men and four women; three current smokers, one ex-smoker, four non-smokers) of mean (SD) age 52.4 (16.6) years (range 33-75) were enrolled in the study. None had associated CVD. The diagnosis was established histopathologically by VATS in six patients and by transbronchial lung biopsy in two. The mean (SD) \%VC was 85.1 (20.8)\% (range 48.7-111.1) and mean $\mathrm{PaO}_{2}$ on room air breathing was 10.9 (1.3) kPa (range 9.2-12.8).

\section{Sarcoidosis}

Ten patients with sarcoidosis with pulmonary lesions (three men and seven women; one current smoker, two ex-smokers, seven non-smokers) of mean (SD) age 44.3 (18.9) years (range 23-75) were enrolled in the study. The diagnosis was clinically established, with pathological findings of noncaseous epithelioid cell granulomas by VATS in one patient, by transbronchial lung biopsy in six patients, and by scalene node biopsy in one. The mean serum angiotensin converting enzyme (ACE) level was 23.0 (13.1) IU/l (range 9.9-47.1) and the mean serum lysozyme level was $16.6(9.9) \mu \mathrm{g} / \mathrm{ml}$ (range 7.5-36.6). The mean (SD) \%VC was 95.0 (15.4)\% (range 71.3117.1 ) and the mean $\mathrm{PaO}_{2}$ on room air breathing was 11.6 (0.9) kPa (range 10.2-13.6).

Diffuse panbronchiolitis (DPB)

Eight patients with DPB (three men and five women; one current smoker, two ex-smokers, five non-smokers) of mean (SD) age 53.3 ( 14.0 ) years (range 33-74) were enrolled. All patients satisfied the diagnostic criteria for DPB published by the Japanese Ministry of Health and Welfare, ${ }^{25}$ and the diagnosis was also pathologically confirmed by OLB. The mean \%VC was 86.8 (15.4)\% (range 64.4-107.7) and the mean $\mathrm{PaO}_{2}$ on room air breathing was 9.8 (1.3) kPa (range 8.3-11.8).

There were no significant differences in the mean \%VC and $\mathrm{PaO}_{2}$ between the above groups. All healthy volunteers had normal chest radiographs, were free of symptoms, and not taking any medications

\section{Blood sample collection and bronchoalveolar lavage} Informed consent was obtained from all patients and healthy volunteers, and both serum and BAL fluid samples were obtained from all subjects. Peripheral venous blood samples were taken immediately after hospital admission and the serum was stored at $-80^{\circ} \mathrm{C}$ until use. BAL was performed as described previously ${ }^{26}$ using a flexible fibreoptic bronchoscope (Olympus 1T-200, Olympus, Tokyo, Japan) after local anaesthesia of the upper airway with $4 \%$ lidocaine. Briefly, the bronchoscope was wedged for lavage into one of the subsegmental bronchi of the right middle lobe or, in patients with peripheral opacities, into areas of lung parenchyma otherwise normal on the chest radiograph. BAL was performed four times using $50 \mathrm{ml}$ aliquots of sterile physiological saline solution at body temperature.

\section{Total and differential cell counts of BAL fluid}

The BAL fluid was passed through two sheets of gauze and then centrifuged at $500 \mathrm{~g}$ for 10 minutes at $4^{\circ} \mathrm{C}$. The remaining fluid was centrifuged at $500 \mathrm{~g}$ for 5 minutes and the supernatant was stored at $-80^{\circ} \mathrm{C}$ for further quantification of non-cellular components. After washing twice with phosphate buffered saline solution (PBS), cells were suspended with 10\% heat inactivated fetal calf serum and counted using a haemocytometer. Differential cell counts were determined from cell suspensions displayed on slides using a cytocentrifuge (Cytospin 2; Shandon Instruments; Sewickley, PA, USA). The cells were dried, fixed on the slide, and then stained by the May-Grunwald-Giemsa method. Two hundred cells were identified under a photomicroscope. Subsets of lymphocytes in BAL fluid were examined by direct immunofluorescence staining using fluorescein isothiocyanate labelled murine monoclonal anti-CD4 and anti-CD8 antibodies (Becton Dickinson; Mountain View, CA, USA). The stained cells were analysed on a flow cytometer (FACScan; Becton Dickinson, FACS Division), and a computer system (Consort 30; Becton Dickinson) was used for data acquisition and analysis.

\section{Measurement of SP-A, SP-D, KL-6, SLX, and CA19-9} levels in the serum and BAL fluid

The level of each marker was measured by commercially available specific kits according to the protocols provided by 

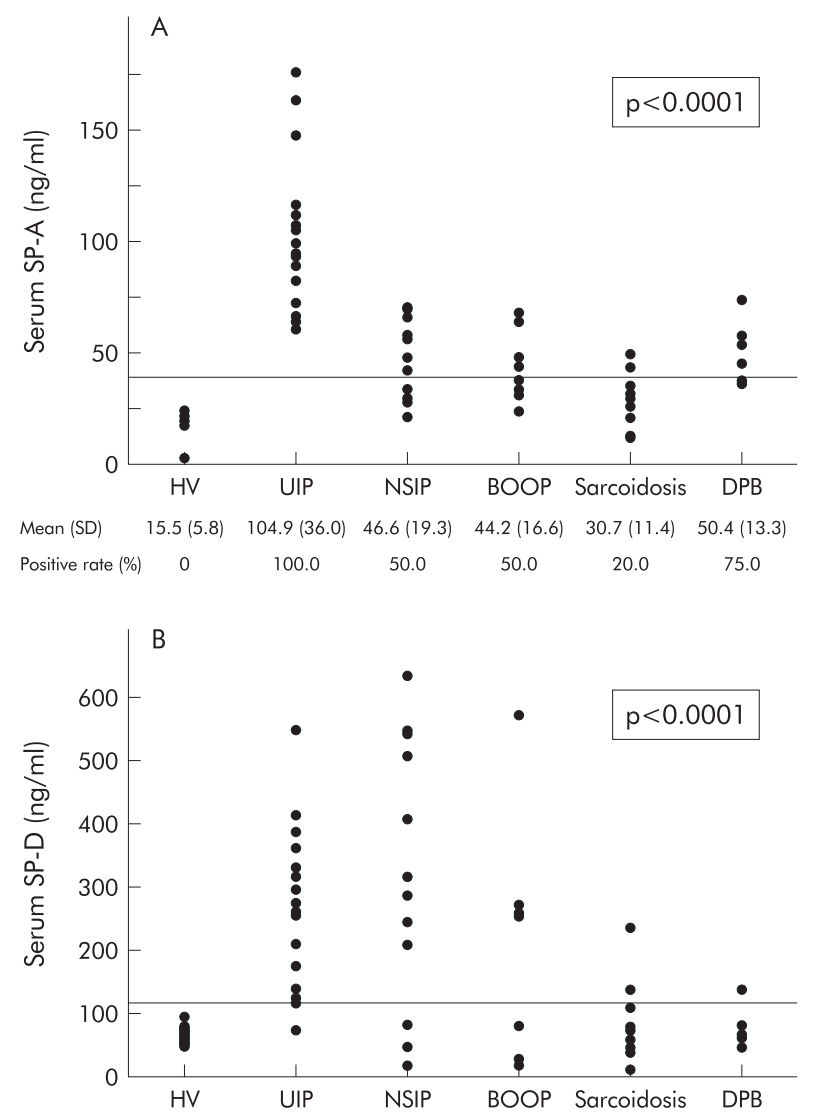

Mean (SD) $\quad 66.3$ (13.5) $259.6(122.4) 310.3(201.5) \quad 190.9(179.0) \quad 86.6(61.1) \quad 77.2(25.0)$ $\begin{array}{lllllll}\text { Positive rate (\%) } & 0 & 93.8 & 75.0 & 50.0 & 20.0 & 12.5\end{array}$

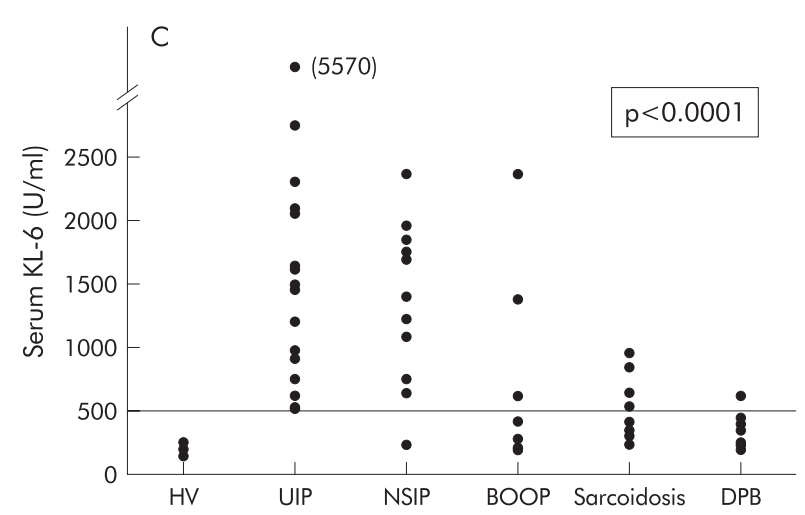

Mean (SD) $\quad 162.2(31.6) \quad 1462(1161) \quad 1185$ (589) 762.9 (722.1) $437.9(233.5) \quad 300.8$ (127.5)

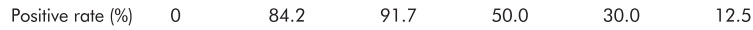

Figure 1 Serum concentrations of (A) SP-A, (B) SP-D, and (C) KL-6 in patients with various lung diseases and healthy volunteers. The cut off values (dotted lines) for these antigens were set at $43.8 \mathrm{ng} / \mathrm{ml}$ for SP-A, $110 \mathrm{ng} / \mathrm{ml}$ for SP-D, and $500 \mathrm{U} / \mathrm{ml}$ for KL-6. Positive rates: percentage of subjects with values above the cut off level of each protein. $p$ values for the overall comparison of all six subject groups are given. $\mathrm{SD}=$ standard deviation; $\mathrm{HV}=$ healthy volunteers; $U \mathrm{IP}=$ usual interstitial pneumonia; NSIP=non-specific interstitial pneumonia; $\mathrm{BOOP}=$ bronchiolitis obliterans organising pneumonia; $\mathrm{DPB}=$ diffuse panbronchiolitis.

the manufacturer. SP-A levels were measured by a sandwichtype enzyme immunoassay (EIA) kit (SP-A test-F; Kokusai Shiyaku Co, Hyogo, Japan); SP-D concentrations were also measured by a sandwich-type EIA kit (SP-D kit Yamasa; Yamasa Shoyu Co, Tokyo); KL-6 concentrations were measured by a sandwich-type electrochemiluminescence immunoassay (ECLIA) kit (Picolumi KL-6; Sanko Junyaku Co,
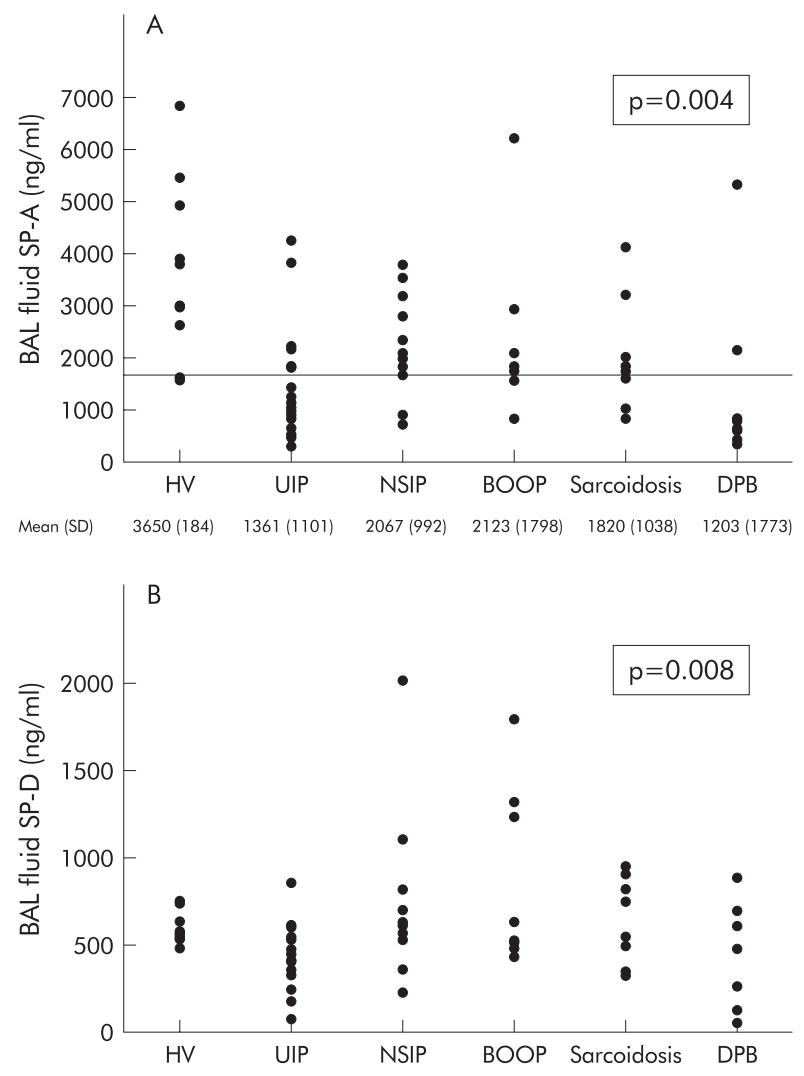

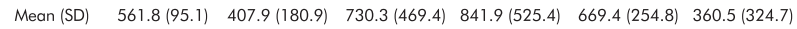

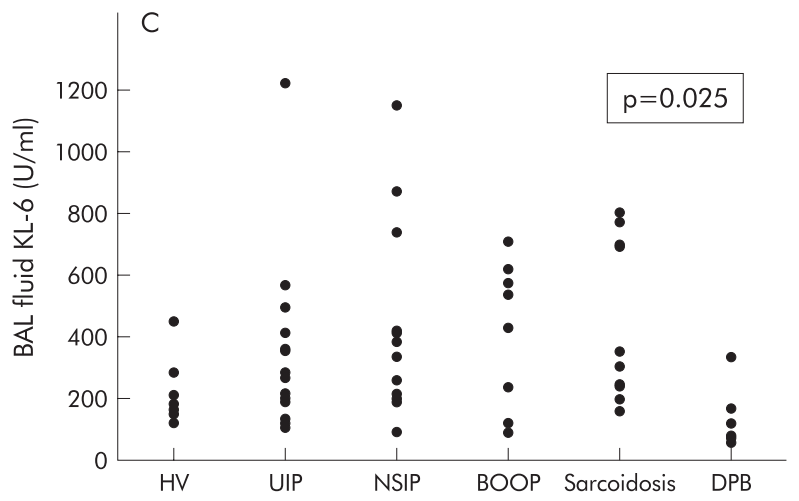

$\begin{array}{lllllll}\text { Mean (SD) } \quad 189.2(105.3) & 307.9(257.5) & 427.2(323.2) & 403.9(238.9) & 434.5(266.4) & 114.8(88.9)\end{array}$

Figure 2 BAL fluid concentrations of (A) SP-A, (B) SP-D, and (C) $\mathrm{KL}-6$ in patients with various lung diseases and healthy volunteers. $\mathrm{P}$ values for the overall comparison of all six subject groups are given. For abbreviations, see legend to fig 1 .

Tokyo); SLX concentrations were measured by a radioimmunoassay (RIA) kit (SLX Otsuka; Otsuka Pharmaceutical Co, Tokyo); and CA19-9 concentrations were also measured by an RIA kit (CA19-9 RIA kit; TFB Co, Tokyo). Serum cut off values were set at $43.8 \mathrm{ng} / \mathrm{ml}$ for SP-A, $110 \mathrm{ng} / \mathrm{ml}$ for SP-D, $500 \mathrm{U} / \mathrm{ml}$ for KL-6, $38 \mathrm{U} / \mathrm{ml}$ for SLX, and $37 \mathrm{U} / \mathrm{ml}$ for CA19-9.

\section{Statistical analysis}

All values were expressed as mean (SD) or range. Differences between multiple groups were compared by one-way analysis of variance. The post hoc test used was Fisher's PLSD test. We also used the Spearman's rank correlation analysis to examine the relationship between the levels of each marker. Statistical analysis was performed using StatView-J 4.5 software (Abacus Concepts; Berkeley, CA). Statistical significance was defined by a $\mathrm{p}$ value of $<0.05$. 
Table 2 SLX and CA19-9 levels in serum and bronchoalveolar lavage (BAL) fluid (U/ml)

\begin{tabular}{lrlllr}
\hline & $\mathbf{n}$ & $\begin{array}{l}\text { Serum SLX } \\
\text { (positive rate) }\end{array}$ & $\begin{array}{l}\text { Serum CA19-9 } \\
\text { (positive rate) }\end{array}$ & $\begin{array}{l}\text { BAL fluid } \\
\text { SLX* }\end{array}$ & $\begin{array}{l}\text { BAL fluid } \\
\text { CA19-9** }\end{array}$ \\
\hline Patients & 57 & & & & \\
UIP & 19 & $35.5(16.2) ;(33.3 \%)$ & $44.0(78.7) ;(27.8 \%)$ & $103.7(142.6)$ & $294.0(792.0)$ \\
NSIP & 12 & $33.8(6.8) ;(25.0 \%)$ & $50.4(97.3) ;(25.0 \%)$ & $100.9(161.4)$ & $159.8(338.4)$ \\
BOOP & 8 & $37.8(14.4) ;(50.0 \%)$ & $27.9(31.7) ;(25.0 \%)$ & $107.3(99.5)$ & $137.5(202.5)$ \\
Sarcoidosis & 10 & $29.5(7.3) ;(20.0 \%)$ & $18.8(17.3) ;(20.0 \%)$ & $31.0(11.7)$ & $60.3(62.0)$ \\
DPB & 8 & $46.0(23.4) ;(62.5 \%)$ & $66.1(60.8) ;(62.5 \%)$ & $300.6(304.4)$ & $1506.0(2436.6)$ \\
Healthy volunteers & 9 & $29.3(4.6) ;(0 \%)$ & $12.9(8.5) ;(0 \%)$ & $21.2(8.3)$ & $33.2(63.0)$ \\
\hline
\end{tabular}

Data are expressed as mean (SD)

UIP=usual interstitial pneumonia; NSIP=non-specific interstitial pneumonia; $B O O P=$ bronchiolitis obliterans organising pneumonia; $\mathrm{DPB}=$ diffuse panbronchiolitis.

${ }^{*} \mathrm{p}=0.005$ for the overall comparison of all six subject groups; ${ }^{*} \mathrm{p}=0.02$ for the overall comparison of all six subject groups.

\section{RESULTS \\ Differential cell count of BAL fluid}

Table 1 shows the characteristics of cells in the BAL fluid of all subjects. The percentage of macrophages was significantly higher in patients with UIP than in those with NSIP, BOOP, sarcoidosis $(p<0.05)$, and DPB $(p<0.0001)$. The percentage of lymphocytes in patients with UIP was lower than in those with NSIP $(p=0.0003)$, sarcoidosis $(p<0.0001)$, and BOOP $(p=0.005)$, and in patients with DPB the percentage of lymphocytes was also significantly lower than in those with NSIP $(p=0.0004)$, BOOP $(p=0.004)$, and sarcoidosis $(p<0.0001)$. The percentage of macrophages in patients with DPB was significantly lower than in those with NSIP, sarcoidosis $(p<0.0001)$, and BOOP $(p=0.0001)$, while the percentage of neutrophils was significantly the highest of all six subject groups $(\mathrm{p}<0.0001)$. The $\mathrm{CD} 4 / \mathrm{CD} 8$ ratio in lymphocyte subsets in patients with sarcoidosis was significantly higher than all other subject groups (healthy volunteers, $\mathrm{p}=0.004$; UIP, $\mathrm{p}=0.001$; NSIP, $\mathrm{p}<0.0001$; BOOP, $\mathrm{p}=0.0004 ; \mathrm{DPB}, \mathrm{p}=0.0006$ ).

\section{Serum levels of SP-A, SP-D and KL-6}

The mean serum concentrations of SP-A, SP-D, and KL-6 of nine healthy volunteers were lower than the cut off values (fig $\mathrm{IA}-\mathrm{C}$ ). As shown in fig $1 \mathrm{~A}$, the serum levels of SP-A were significantly higher in patients with UIP $(p<0.0001)$, NSIP, DPB $(p=0.003)$, and BOOP $(p<0.05)$ than in healthy volunteers. The mean serum concentration of SP-A in patients with UIP was significantly the highest among patients with lung diseases $(p<0.0001$, respectively $)$. Moreover, the serum concentrations of SP-A were higher than the cut off level in all patients with UIP (fig 1A). The serum concentrations of SP-D were significantly higher in patients with UIP $(\mathrm{p}=0.0006)$ and NSIP $(p<0.0001)$ than in healthy volunteers, and those of patients with UIP and NSIP were significantly higher than the concentrations in patients with sarcoidosis (UIP, $p=0.001$; NSIP, $p=0.0001$ ) and DPB (UIP, $p=0.002$; NSIP, $p=0.0002$ ). The mean serum concentration of KL- 6 was also significantly higher in patients with UIP and NSIP than in healthy volunteers (UIP, $p<0.0001$; NSIP, $p=0.003$ ), and that of patients with UIP was significantly higher than the other disease groups (BOOP, $p<0.05$; DPB, $p=0.0004$; sarcoidosis, $\mathrm{p}=0.0007$ ) except NSIP. The serum concentration of KL-6 in patients with NSIP was significantly higher than in those with DPB $(\mathrm{p}<0.05$, fig $1 \mathrm{C})$.

\section{BAL fluid concentrations of SP-A, SP-D and KL-6}

As shown in fig 2A, BAL fluid concentrations of SP-A were significantly lower in patients with UIP $(p=0.0001)$, NSIP, BOOP $(p<0.05)$, sarcoidosis $(p=0.005)$, and DPB $(p=0.0006)$ than in healthy volunteers, but the concentrations were not significantly different between patients with different lung diseases. BAL fluid concentrations of SP-D were significantly lower in patients with UIP and DPB than in those with NSIP (UIP, $\mathrm{p}=0.009$; DPB, $\mathrm{p}<0.05$ ), BOOP (UIP, $\mathrm{p}=0.002$, DPB, $p=0.004)$, and sarcoidosis $(p<0.05$; fig $2 B)$. The mean $B A L$ fluid concentration of KL-6 was significantly higher in patients with NSIP and sarcoidosis than in controls $(\mathrm{p}<0.05$, respectively) and in those with DPB (NSIP, $p=0.006$; sarcoidosis, $\mathrm{p}=0.007$; fig $2 \mathrm{C}$ ).

SLX and CA19-9 concentrations in serum and BAL fluid The mean (SD) serum concentrations of SLX and CA19-9 in nine healthy volunteers were 29.3 (4.6) and 12.9 (8.5) U/ml, respectively, and the levels in each subject were below the cut off values. As shown in table 2, there was no significant difference in serum and BAL fluid concentrations of SLX and CA19-9 between patients with different lung diseases, while BAL fluid concentrations of SLX and CA19-9 in patients with DPB were significantly the highest of all the subject groups.

\section{Correlations between serum markers and BAL fluid markers}

Serum KL-6, SP-A, SLX and CA19-9 concentrations of all subjects correlated significantly with the respective markers in BAL fluid (KL-6, $r=0.451, p=0.0003 ;$ SP-A, $r=-0.330$, $\mathrm{p}=0.008 ; \quad$ SLX $, \quad r=0.344, \mathrm{p}=0.008 ; \quad$ CA19-9, $r=0.723$, $\mathrm{p}<0.0001)$ with the exception of SP-D concentrations $(r=-0.087, \mathrm{p}=0.492)$. Further analysis of data of the entire population (normal subjects + patients with lung diseases) showed significant correlations between serum concentrations of SP-A and serum KL-6 $(r=0.627, \mathrm{p}<0.0001)$, serum SP-D and serum KL-6 $(r=0.628, \mathrm{p}<0.0001)$, serum SLX and serum KL-6 $(r=0.299, \mathrm{p}=0.020)$, serum SP-D and serum SP-A $(r=0.498, \mathrm{p}=0.0001)$, and serum CA19-9 and serum SLX $(r=0.468, \mathrm{p}=0.0003)$. Likewise, analysis of marker concentrations in BAL fluid showed significant correlations between SP-A and KL-6 $(r=0.257, \mathrm{p}=0.038)$, SP-D and KL-6 $(r=0.430$, $\mathrm{p}=0.0005)$, SP-D and SP-A $(r=0.386, \mathrm{p}=0.002)$, SLX and SP-A $(r=-0.407, \mathrm{p}=0.001)$, and CA19-9 and SLX $(r=0.599$, $\mathrm{p}<0.0001)$.

\section{DISCUSSION}

Histopathological findings consistent with UIP are required for the clinical diagnosis of IPF. ${ }^{7-10}$ IPF is the most common idiopathic interstitial pneumonia and the one with the worst prognosis. ${ }^{4-9}$ NSIP is pathologically characterised by interstitial inflammatory cell infiltration with or without fibrosis, and the most characteristic finding in NSIP is the lack of temporal heterogeneity, which is a cardinal feature of UIP. ${ }^{1-7}$ Because patients with pneumonia that clinically mimics UIP such as NSIP have a better prognosis than patients with UIP, surgical lung biopsy is a very important examination for the pathological diagnosis and clinical choice of therapeutic 
management. ${ }^{3-10}$ However, it is difficult to perform this invasive examination in all patients with ILD. Clinicians often speculate on the presence of such pathological changes based on non-invasive imaging studies such as high resolution computed tomographic (HRCT) scans. However, HRCT scans are expensive and do not always discriminate accurately between NSIP and UIP. In the present study we therefore compared the diagnostic value of five biomarkers, SP-A, SP-D, KL-6, SLX, and CA19-9, as less invasive and low cost auxiliary methods to use in combination with HRCT for assessment of patients with ILD.

The major finding was that the serum levels of SP-A, SP-D, and KL-6 in patients with ILD were significantly higher than in healthy volunteers. The serum levels of SP-A in patients with UIP were significantly higher than in patients with NSIP, and BAL fluid levels of SP-D in patients with UIP were significantly lower than in patients with NSIP (figs 1 and 2). These results confirm the findings of previous reports that SP-A, SP-D, and KL-6 are useful markers for the diagnosis of ILD, ${ }^{11-14} 22-24$ and suggest that serum SP-A levels may be particularly useful for discriminating between UIP and other types of interstitial pneumonia such as NSIP. However, as relatively small numbers of patients were used in this study and there was some overlap in the serum SP-A levels between the UIP and NSIP groups, it is still difficult using only serum or BAL fluid markers to discriminate between these two groups without invasive methods.

SP-A and SP-D belong to the collectin subgroup of the C-type lectin superfamily, along with mannose-binding lectin and collectin-43. ${ }^{12}$ They are produced by two types of non-ciliated epithelial cells in the peripheral airway, Clara cells, and alveolar type II cells. KL-6 is a mucin-like high molecular weight glycoprotein and is expressed on type II pneumocytes and respiratory bronchiolar epithelial cells in the normal lung. ${ }^{22-24}$ Proliferating regenerating type II pneumocytes in pneumonitis, such as IPF and radiation pneumonitis, express KL-6 more strongly than normal type II cells. ${ }^{22}$ Several investigators have shown the usefulness of these biomarkers in patients with diffuse pulmonary diseases, particularly ILD. ${ }^{22-24}{ }^{27-29}$ Takahashi et al ${ }^{12}$ reported that assays of serum SP-A and SP-D may assist in making a clinical choice for therapeutic management of patients with IPF. In another study they reported that the assay of serum SP-D was a clinically useful tool for detecting ILD complicated by progressive systemic sclerosis. ${ }^{30}$ They also showed that the levels of SP-A and SP-D in patients with IPF who died within 3 years were significantly higher than in patients who were still alive after 3 years, and that high levels of SP-D are involved in subsequent declines in \%VC and \% TLC. $^{12}$ This may be consistent with the present finding of higher serum SP-A levels in patients with UIP than in patients with NSIP. The patients with IPF in the study by Takahashi $e t$ a $l^{30}$ may have included other types of interstitial pneumonia such as NSIP since they were not necessarily diagnosed by surgical lung biopsy.

When the results of all the subjects in the present study were analysed, a significant correlation was found between both serum levels of SP-A and SP-D and BAL fluid levels of these two surfactants. Furthermore, there was no significant difference in serum levels of SP-D between patients with UIP and NSIP, while a significant difference was found in serum SP-A levels between these two patient groups (fig 1). This suggests that the mechanisms of increased levels of the two proteins could be partly different from each other. According to the reports of Takahashi et al, ${ }^{12}{ }^{30}$ the accelerated production of SP-A and SP-D by type II pneumocytes and destruction of the epithelium-endothelium barrier are likely to be the main causes of the appearance of SP-A and SP-D in the bloodstream, and SP-D leaks into the bloodstream from the alveoli more easily than SP-A because of its solubility. McCormack and colleagues ${ }^{15}$ also reported that the low levels of SP-A in BAL fluid of patients with IPF compared with healthy sub- jects might be the result of reduced access to the alveolar compartment, reduced production of surfactant by the damaged alveolar epithelium, or increased uptake and degradation by macrophages and type II pneumocytes. However, the exact mechanism of clearance of SP-A and SP-D remains unclear, and we could not clarify this difference.

Like SP-A, SP-D and KL-6, it has been also reported that the serum and BAL fluid concentrations of SLX and CA19-9, which are carbohydrate antigens, are raised in patients with IPF, ILD associated with CVD, and DPB. ${ }^{16202131}$ Obayashi et al ${ }^{31}$ showed that BAL fluid concentrations of CA19-9 correlated with other markers of inflammation including elastase, hepatocyte growth factor, and lactic acid dehydrogenase in patients with IPF, and that CA19-9 had a chemotactic activity for neutrophils. In this study, however, there were no significant differences in the serum levels of SLX and CA19-9 between all the groups. BAL fluid concentrations of SLX and CA19-9 only in patients with DPB were significantly the highest of all the patients with lung disease and healthy volunteers. In addition, there were no significant differences in either serum or BAL fluid levels of KL-6 between patients with UIP or NSIP, while serum levels of KL-6 in patients with ILD were significantly higher than in controls. These results suggest that KL-6, SLX, and CA19-9 may be less useful for discriminating between these diseases than SP-A and SP-D.

In conclusion, we found a significantly higher level of SP-A in the serum of patients with UIP than in those with other interstitial pneumonias such as NSIP. However, further studies in a larger number of patients are required to determine the cut off levels of SP-A necessary for diagnosis, as well as prospective studies. SP-A in serum may be a possible candidate for a less invasive biomarker to discriminate between UIP and NSIP.

\section{ACKNOWLEDGEMENT}

The authors thank Dr M Kitaichi (Department of Laboratory Medicine, Kyoto University Hospital) for the valuable advice regarding pathological diagnosis.

\section{Authors' affiliations}

H Ishii, H Mukae, H Kaida, T Nagata, S Kohno, Second Department of Internal Medicine, Nagasaki University School of Medicine, Nagasaki, Japan

J Kadota, Second Department of Internal Medicine, Oita Medical

University, Oita, Japan

K Abe, S Matsukura, Third Department of Internal Medicine, Miyazaki Medical College, Miyazaki, Japan

\section{REFERENCES}

1 Katzenstein AA, Fiorelli RF. Nonspecific interstitial pneumonia/fibrosis. Histologic features and clinical significance. Am J Surg Pathol 1994:18:136-47.

2 Travis WD, Matsui K, Moss J, et al. Idiopathic nonspecific interstitial pneumonia: Prognostic significance of cellular and fibrosing patterns: survival comparison with usual interstitial pneumonia and desquamative interstitial pneumonia. Am J Surg Pathol 2000;24:19-33.

3 Katzenstein AA, Myers JL. Nonspecific interstitial pneumonia and the other idiopathic interstitial pneumonias: classification and diagnostic criteria. Am J Surg Pathol 2000;24: 1-3.

4 Daniil ZD, Gilchrist FC, Nicholson AG, et al. A histologic pattern of nonspecific interstitial pneumonia is associated with a better prognosis than usual interstitial pneumonia in patients with cryptogenic fibrosing alveolitis. Am J Respir Crit Care Med 1999;160:899-905

5 Cottin V, Donsbeck AV, Revel D, et al. Nonspecific interstitial pneumonia: Individualization of a clinicopathologic entity in a series of 12 patients. Am J Respir Crit Care Med 1998;158:1286-93.

6 Nagai S, Kitaichi $M$, Itoh $H$, et al. Idiopathic nonspecific interstitial pneumonia/fibrosis: Comparison with idiopathic pulmonary fibrosis and BOOP. Eur Respir J 1998;12:1010-19.

7 Myers JL. NSIP, UIP, and the ABCs of idiopathic interstitial pneumonias. Eur Respir J 1998;12:1003-4.

8 Bjoraker JA, Ryu JH, Edwin MK, et al. Prognostic significance of histopathologic subsets in idiopathic pulmonary fibrosis. Am J Respir Crit Care Med 1998;157:199-203.

9 Katzenstein AA, Myers JL. Idiopathic pulmonary fibrosis: Clinical relevance of pathologic classification. Am J Respir Crit Care Med $1998 ; 157: 1301-15$. 
10 Hunninghake GW, Zimmerman MB, Schwartz DA, et al. Utility of a lung biopsy for the diagnosis of idiopathic pulmonary fibrosis. Am J Respir Crit Care Med 2001;164:193-6.

11 Shijubo N, Honda Y, Itoh Y, et al. BAL surfactant protein A and Clara cell 10-kDa protein levels in healthy subjects. Lung 1998;176:257-65.

12 Takahashi $\mathbf{H}$, Fujishima T, Koba H, et al. Serum surfactant proteins A and $D$ as prognostic factors in idiopathic pulmonary fibrosis and their relationship to disease extent. Am J Respir Crit Care Med 2000;162:1109-14

13 Honda $Y$, Kuroki $Y$, Matsuura E, et al. Pulmonary surfactant protein D in sera and bronchoalveolar lavage fluids. Am J Respir Crit Care Med $1995 ; 152: 1860-6$

14 Kuroki Y, Takahashi H, Chiba H, et al. Surfactant proteins A and D: disease markers. Biochim Biophys Acta 1998;1408:334-45.

15 McCormack FX, King TE, Jr., Bucher BL, et al. Surfactant protein A predicts survival in idiopathic pulmonary fibrosis. Am J Respir Crit Care Med 1995;152:751-9.

16 Yokoyama A, Kohno N, Kondo K, et al. Comparative evaluation of sialylated carbohydrate antigens, KL-6, CA19-9 and SLX as serum markers for interstitial pneumonia. Respirology 1998;3:199-202.

17 Kannagi R, Fukushi Y, Tachikawa T, et al. Quantitative and qualitative characterization of human cancer-associated serum glycoprotein antigens expressing fucosyl or sialyl-fucosyl type 2 chain polylactosamine. Cancer Res 1986;46:2619-26

18 Magnani JL, Steplewski Z, Koprowski H, et al. Identification of the gastrointestinal and pancreatic cancer-associated antigen detected by monoclonal antibody $19-9$ in the sera of patients as a mucin. Cancer Res 1983;43:5489-92.

19 Kohno N, Akiyama M, Kyoizumi S, et al. Detection of soluble tumor-associated antigens in sera and effusions using novel monoclonal antibodies, KL-3 and KL-6, against lung adenocarcinoma. Jpn J Clin Oncol 1985; 18:203-16
20 Sato $\mathrm{H}$, Kamma H, Ogata $\mathrm{T}$, et al. Clinical significance of serum levels of a carbohydrate antigen, sialyl SSEA-1, in patients with fibrosing lung disease. Am Rev Respir Dis 1991;144:1177-81.

21 Mukae H, Hirota M, Kohno S, et al. Elevation of tumor-associated carbohydrate antigens in patients with diffuse panbronchiolitis. Am Rev Respir Dis 1993; 148:744-51.

22 Kohno N, Kyoizumi S, Awaya Y, et al. New serum indicator of interstitial pneumonitis activity: Sialylated carbohydrate antigen KL-6. Chest 1989;96:68-73.

23 Kobayashi J and Kitamura S. KL-6: A serum marker for interstitial pneumonia. Chest 1995; 108:311-5.

24 Kohno N, Awaya Y, Oyama T, et al. KL-6, a mucin-like glycoprotein, in bronchoalveolar lavage fluid from patients with interstitial lung disease. Am Rev Respir Dis 1993;148:637-42.

25 Homma H, Yamanaka A, Tanimoto S, et al. Diffuse panbronchiolitis: a disease of the transitional zone of the lung. Chest 1983;83:63-9.

26 Mukae H, Kadota J, Kohno S, et al. Increase in activated CD8+ cells in bronchoalveolar lavage fluid in patients with diffuse panbronchiolitis. Am J Respir Crit Care Med 1995;152:613-8.

27 Kubo M, Ihn H, Yamane K, et al. Serum KL-6 in adult patients with polymyositis and dermatomyositis. Rheumatology 2000;39:632-6.

28 Nakajima $\mathbf{H}$, Harigai $M$, Hara $M$, et al. KL-6 as a novel serum marker for interstitial pneumonia associated with collagen diseases. J Rheumatol 2000:27: 1 164-70.

29 Fukaya S, Oshima H, Kato K, et al. KL-6 as a novel serum marker for activities of interstitial pneumonia in connective tissue diseases. Rheumatol Int 2000;19:223-5.

30 Takahashi H, Kuroki Y, Tanaka H, et al. Serum levels of surfactant proteins $A$ and $D$ are useful biomarkers for interstitial lung disease in patients with progressive systemic sclerosis. Am J Respir Crit Care Med 2000; 162:258-63.

31 Obayashi Y, Fujita J, Nishiyama T, et al. Role of carbohydrate antigens sialyl Lewis (a) (CA 19-9) in bronchoalveolar lavage in patients with pulmonary fibrosis. Respiration 2000;67:146-52.

LUNG ALERT

Traffic related air pollutants shorten life expectancy

$\Delta$ Hoek G, Brunekref B, Goldbohm S, et al. Association between mortality and indicators of traffic-related air pollution in the Netherlands: a cohort study. Lancet 2002;360:1203-9.

his was a study of a random sample of 5000 subjects aged 59-69 years selected from the Netherlands Cohort Study on Diet and Cancer. The patients were contacted every 2 years from 1986 to 1994 to determine migration and to assess vital status and deaths. There were 489 deaths in this period. Cardiopulmonary mortality was associated with living near a major roadway-that is, $100 \mathrm{~m}$ from a highway or $50 \mathrm{~m}$ from a main road (relative risk $1.95,95 \%$ CI 1.09 to 3.52 ).

This paper is yet further evidence that air pollutants or some closely associated pollutant from road traffic contribute to cardiopulmonary mortality.

A Bhowmik

angshub@altavista.ne 